\title{
La FMH en 2009
}

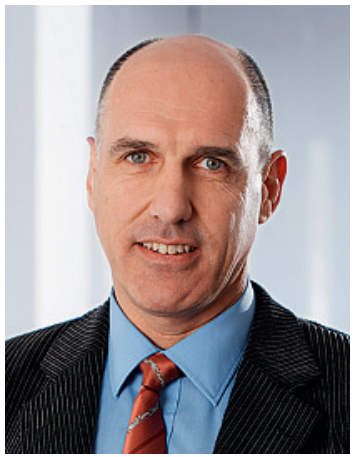

Jacques de Haller
Le Comité central de la FMH, puis l'Assemblée des délégués, ont défini l'automne dernier un certain nombre d'objectifs stratégiques qui ont été adoptés par la Chambre médicale du 11 décembre dernier.

Si les enjeux qui se cachent derrière ces objectifs comme certains aspects de la formation, ou certaines questions tarifaires - sont parfois très «sectoriels», l'ensemble constitue un vaste champ d'action qui recouvre largement les intérêts de la profession. Le Comité central porte une très grande attention à ce que la FMH représente bien l'ensemble de ses membres, l'ensembledu corps médical. Vous pouvez retrouver l'énoncé de ces objectifs stratégiques de la FMH à l'adresse www.fmh.ch $\rightarrow$ Qui sommes-nous? $\rightarrow$ Le Comité central de la FMH $\rightarrow$ Stratégie du Comité central de la FMH.

Il est important en ce début d'année de voir comment ces intentions vont s'exprimer dans les faits, comment les mots vont se traduire en actions. Car il y a plusieurs domaines dans lesquels l'actualité devient chaude, plus que nous ne l'aurions souhaité.

En tête de ces sujets chauds, le laboratoire. C'est malheureusement une vraie provocation de la part du chef du Département fédéral de l'intérieur que de vouloir toucher au laboratoire du cabinet, sujet-phare de la fameuse manifestation du $1^{\text {er }}$ avril 2006 et thème de ralliement par excellence des médecins de premier recours.

Face à la rigidité obtuse de l'administration fédérale et à un certain nombre de mensonges inacceptables (voir l'article d'Ernst Gähler en p. 7), la FMH a d'une part préparé une contre-proposition pour laquelle nous nous battrons (tarif «Point of Care»), mais elle s'est aussi préparée ces dernières semaines à tout un éventail de mesures de lutte vigoureuses, de divers ordres, qui sont maintenant prêtes à être mises en œuvre dans les mois qui viennent, selon les besoins et l'évolution de la situation, avec votre aide à toutes et à tous.

Et puis aux côtés du laboratoire viennent toute une série d'autres domaines importants, même s'ils sont moins «médiatisés», et que faute de place on ne peut qu'évoquer brièvement ici mais sur lesquels nous reviendrons bien sûr:

- sur le plan tarifaire encore, la valeur du point pour l'assurance-accident, de même que la réduction de la marge pour la pro-pharmacie;

- les DRG et les mesures que nous prenons pour en assurer une introduction correcte et sûre, sans atteinte à la qualité des prestations;
- la garantie de qualité, justement, dans les cabinets aussi, avec le souci de garder en nos mains les éléments, les définitions, les critères qui doivent continuer à nous appartenir;

- la formation, avec la préparation des nouvelles procédures d'accréditation, travail immense et coûteux, et celle du livret de formation;

- tout le domaine de la prévention, avec la préparation d'une nouvelle loi (enfin!) mais aussi avec le combat engagé par les milieux économiques contre les mesures de promotion de la santé - la FMH va devoir là s'engager fermement en faveur des «patients-consommateurs»;

- l'informatisation des cabinets médicaux enfin; la carte d'assuré a été repoussée à 2010, mais la nouvelle carte de membre de la FMH reste prévue pour 2009, avec sa puce électronique et ses multiples possibilités d'applications sécurisées.

L'engagement de la FMH dans le secteur «eHealth» ira dans le sens du respect de la pratique médicale, dans ce qu'elle a et doit garder de plus humain: préserver la relation avec les patients, protection des données, et sans oublier évidemment que l'informatisation ne doit en aucun cas être une charge supplémentaire mais bien une facilitation.

Et puis reste bien sûr la révision de la LAMal, LAMal qui sert de cadre légal à l'essentiel des points évoqués ci-dessus. Ce cadre légal doit à tout prix rester adéquat, centré sur les besoins des patient-e-s et de la médecine - la médecine de qualité telle que nous la comprenons!

Pas question donc de laisser basculer tout le système de santé dans les mains d'un seul de ses acteurs, en l'occurrence les assureurs, et de laisser les seules lois du marché déterminer le mode d'accès au soins.

Nous avons là plusieurs importantes propositions, constructives, tant pour définir le cadre du système de santé («Proposition FMH-CDS») que pour permettre son fonctionnement à long terme (entre autres les «Thèses de la FMH sur le Managed Care»); ce sont des propositions respectueuses tant de nos membres, quelle que soit leur orientation professionnelle, que de nos partenaires, et nous allons déjà dans les jours qui viennent recommencer à les défendre dans les cercles politiques, après le forfait du Conseil des Etats le 18 décembre dernier.

La FMH s'est considérablement développée ces dernières années, elle est présente et reconnue très largement. Cela signifie un volume d'activité très augmenté, vous l'aurez vu; mais nous tenons à notre slogan et nous l'assumerons en 2009 aussi: «Pas de politique de la santé sans la FMH»!

Jacques de Haller, président de la FMH 